\title{
Experimental Evaluation of Cooperative Relative Positioning for Intelligent Transportation System
}

\author{
Suhua Tang, ${ }^{1,2}$ Nao Kawanishi, ${ }^{2}$ Rei Furukawa, ${ }^{2,3}$ and Nobuaki Kubo ${ }^{4}$ \\ ${ }^{1}$ Graduate School of Informatics and Engineering, The University of Electro-Communications, 1-5-1 Chofugaoka, Chofu, \\ Tokyo 182-8585, Japan \\ ${ }^{2}$ Advanced Telecommunications Research Institute International, 2-2-2 Hikaridai, Seika-cho, Soraku-gun, Kyoto 619-0288, Japan \\ ${ }^{3}$ Kozo Keikaku Engineering Inc., 4-5-3 Chuo, Nakano-ku, Tokyo 164-0011, Japan \\ ${ }^{4}$ Tokyo University of Marine Science and Technology, 2-1-6 Etchujima, Koto-ku, Tokyo 135-8533, Japan
}

Correspondence should be addressed to Suhua Tang; shtang@uec.ac.jp

Received 12 August 2014; Accepted 1 November 2014; Published 30 November 2014

Academic Editor: Yuei-An Liou

Copyright (C) 2014 Suhua Tang et al. This is an open access article distributed under the Creative Commons Attribution License, which permits unrestricted use, distribution, and reproduction in any medium, provided the original work is properly cited.

Support system for safe driving heavily depends on global navigation satellite system. Pseudoranges between satellites and vehicles are measured to compute vehicles' positions and their relative positions. In urban areas, however, multipath errors (MPEs) in pseudoranges, caused by obstruction and reflection of roadside buildings, greatly degrade the precision of relative positions. On the other hand, simply removing all reflected signals might lead to a shortage of satellites in fixing positions. In our previous work, we suggested solving this dilemma by cooperative relative positioning (CoRelPos) which exploits spatial correlation of MPEs. In this paper, we collected the trace data of pseudoranges by driving cars in urban areas, analyzed the properties of MPEs (specifically, their dependency on signal strength, elevation angles of satellites, and receivers' speeds), and highlighted their spatial correlation. On this basis, the CoRelPos scheme is refined by considering the dynamics of MPEs. Evaluation results under practical vehicular scenarios confirm that properties of MPEs can be exploited to improve the precision of relative positions.

\section{Introduction}

The rapid and wide spread of motor vehicles after World War II has greatly changed human society. On one hand, it frees common people from the limitations of their geography and facilitates remote travel. On the other hand, it also brings some undesirable consequences such as vehicle accidents, which often lead to severe body injuries or even the loss of precious lives. To reduce vehicle accidents, many efforts have been devoted to support systems for safe driving, from two aspects. (i) Each vehicle independently detects the presence of nearby vehicles by using cameras or radars. But this fails to work when the line of sight (LOS) path between vehicles is obstructed. (ii) Vehicles cooperate to measure their distances, which relies on global navigation satellite system (GNSS) [1] and intervehicle communications (IVC) [2]. In this system, each vehicle receives positioning signals from satellites, measures the pseudoranges between itself and satellites, and on this basis computes its own position. Each vehicle further exchanges its own position and moving speed with nearby vehicles via IVC. Based on current positions and moving speeds of two vehicles, their distance (relative position) several seconds later is estimated, and the driver is warned in case a collision is predicted to happen. Such applications have been extensively evaluated in the ASV4 (advanced safety vehicle) experiments in Japan [3]. In our previous work, we found that although the real-time delivery of position information can be realized [4], the precision of position remains a big problem, especially in urban areas. How to improve the precision of relative position is the focus of this paper.

Positioning precision of GNSS might be degraded by several factors: clock and orbital errors of GNSS satellites, propagation delay caused by the atmosphere (ionosphere, troposphere), obstruction and reflection of roadside buildings, and clock error and thermal noise at a GNSS receiver. 
Among these factors, propagation delay due to atmosphere is known to have spatial correlation and can be mitigated by ground based augmentation systems such as DGPS (differential global positioning system) [1] or space-based augmentation systems (SBAS).

Accurately measuring pseudoranges between vehicles and satellites usually requires a LOS path. In urban areas, however, the LOS path might be obstructed by roadside buildings, and then a GNSS receiver receives a reflected signal instead. The extended propagation length of a positioning signal due to reflections, compared with the LOS path, is called a multipath error (MPE). MPE, which only occurs in vehicles but not in reference stations installed in an open sky environment, can hardly be mitigated by DGPS and remains the largest error source in urban areas. Therefore, many efforts have been devoted to remove reflected signals before fixing absolute positions, including antenna design, correlator refinement (narrow correlator [5], early-late slope correlator [6], and strobe correlator [7]), modulation design (binary offset carrier in modern GPS [8]), carrier smoothing (Hatch filter [9]), signal separation (multipath estimating delay lock loop [10], spatial sampling via antenna array [11]), and detection of LOS path (using a 3D GIS database [12] or an omnidirectional infrared camera [13]). Removing all reflected signals, however, might lead to a shortage of satellites in fixing positions because few satellites are directly visible to vehicles in urban canyons.

Relative position between two vehicles plays a more important role than their absolute positions in avoiding collision accidents. In addition, computing relative positions needs the cooperation between vehicles. Vehicles, forming a network, can cooperate to improve positioning precision [14], using both intra- and intervehicle measurements. The latter requires a ranging capability in IVCs, which is absent in vehicular communication protocols such as 802.11p [15]. Instead of directly exchanging position information, it is suggested in [16] that each vehicle exchange its measured pseudoranges with nearby vehicles so that relative position is computed by using positioning signals from common satellites. But how to handle MPEs and how to deal with GNSS outage are left untouched. A GNSS outage occurs when the number of satellites is insufficient for fixing positions. This problem is usually solved by using an inertial navigation system (INS) with a gyroscope and accelerometers [17]. It is also possible to realize a tight integration of GNSS and INS by a single Kalman filter $[18,19]$. As for vehicular networks, a car speed can be estimated from speed pulses instead of using accelerometers.

In our previous work [20], we assumed local spatial correlation of MPEs by the following reasoning: usually higher precision of relative positions is required when intervehicle distances get shorter; on the other hand, nearby receivers have similar spatial propagation environments and their MPEs are correlated; for example, MPEs in the pseudoranges at car $A$ and $\operatorname{car} B$ in Figure 1 are almost the same. On this basis, we extended the DGPS concept and suggested a cooperative relative positioning (CoRelPos) scheme, using correlated information from common satellites, including reflected signals, to accurately compute relative positions. Initial results,

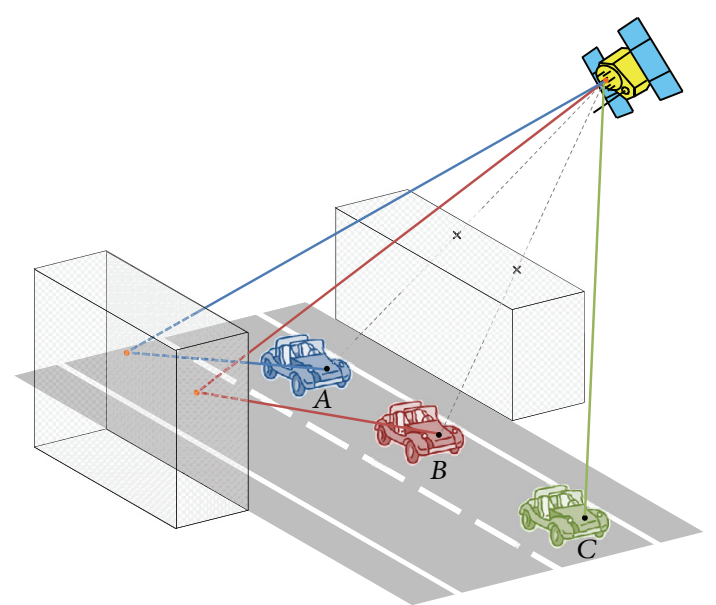

FIgURE 1: Correlation in MPEs of pseudoranges. MPEs at $A$ and $B$ are almost the same and are regarded as being spatially correlated.

where two receivers were installed on stationary cars or on one moving car, were reported.

In this work, we installed GNSS receivers on different cars and collected the trace data of pseudoranges by driving cars in urban areas. Based on these experimental data, we analyzed the properties of MPEs (specifically, their dependency on signal strength, elevation angles of satellites, and receivers' moving speed) and, most importantly, highlighted their spatial correlation. On this basis, the CoRelPos scheme is refined by considering the dynamics of MPEs. Evaluation results under practical vehicular scenarios confirm that properties of MPEs can be exploited to improve the precision of relative positions.

The rest of this paper is organized as follows: Section 2 briefly introduces the CoRelPos scheme [20]. Section 3 describes the experiment setup. Then, experimental results of MPEs are analyzed in Section 4. On this basis, the CoRelPos scheme is refined in Section 5, and positioning results are presented in Section 6. Finally, we conclude the paper with Section 7.

\section{Brief Introduction of the CoRelPos Scheme}

The basic idea of CoRelPos [20] is to use all correlated signals at two vehicles, including reflected ones, in fixing their relative position. To this end, each vehicle measures pseudoranges and exchanges with nearby vehicles via IVCs. Then, each vehicle selects signals with high correlation and uses them to compute relative positions.

A pseudorange $p_{n}^{(s)}$ between satellite $s=k, l$ and receiver $n=a, b$ is expressed as [1]

$$
\begin{aligned}
p_{n}^{(s)}= & \rho_{n}^{(s)}+c \cdot\left(\Delta t_{n}-\Delta T^{(s)}\right)+d_{\mathrm{ion}, n}^{(s)}+d_{\text {trop }, n}^{(s)} \\
& +m_{n}^{(s)}+\varepsilon_{n}^{(s)},
\end{aligned}
$$

where $\rho_{n}^{(s)}$ is the real distance between $s$ and $n, c$ is light speed, $\Delta t_{n}$ and $\Delta T^{(s)}$ are clock errors of $n$ and $s, d_{\text {ion, } n}^{(s)}$ and $d_{\text {trop }, n}^{(s)}$ are the delay effects of ionosphere and troposphere, 
$m_{n}^{(s)}$ is a MPE, and $\varepsilon_{n}^{(s)}$ is the effect of thermal noise. When two receivers are close to each other, common errors $\left(\Delta T^{(s)}\right)$ and spatially correlated errors $\left(d_{\text {ion, } n}^{(s)}, d_{\text {trop }, n}^{(s)}\right)$ are cancelled out by differential operations. Then, the double difference of pseudoranges between two satellites $k, l$ and two receivers $a, b$ can be approximated as

$$
\begin{aligned}
p_{a b}^{(k l)}= & \left(p_{a}^{(k)}-p_{b}^{(k)}\right)-\left(p_{a}^{(l)}-p_{b}^{(l)}\right) \\
\approx & \rho_{a b}^{(k l)}+\left(m_{a}^{(k)}-m_{b}^{(k)}\right)-\left(m_{a}^{(l)}-m_{b}^{(l)}\right) \\
& +\left(\varepsilon_{a}^{(k)}-\varepsilon_{b}^{(k)}\right)-\left(\varepsilon_{a}^{(l)}-\varepsilon_{b}^{(l)}\right) .
\end{aligned}
$$

Assume satellite $l$ has a large elevation angle so its signal is directly received by both receivers without MPEs; that is, $m_{a}^{(l)}=m_{b}^{(l)}=0$. Then, the MPE difference with respect to satellite $k$ can be estimated by

$$
m_{a}^{(k)}-m_{b}^{(k)} \approx p_{a b}^{(k l)}-\rho_{a b}^{(k l)}
$$

$m_{a}^{(k)}$ and $m_{b}^{(k)}$ are regarded as being spatially correlated if $\left|p_{a b}^{(k l)}-\rho_{a b}^{(k l)}\right|$ is less than a threshold. When computing $\rho_{a b}^{(k l)}$, real positions of two receivers are required to find the true distances $\rho_{n}^{(s)}$. In the actual protocol, a Kalman filter [21] is exploited to predict these positions.

The state of a Kalman filter, $\mathbf{X}_{n, t}=\left[\mathbf{r}_{n, t} ; \mathbf{v}_{n, t} ; \dot{\mathbf{v}}_{n, t} ; \delta_{n, t} ; \Delta_{n, t}\right]$ (column vectors are stacked by the operator “;”), consists of receiver position $\mathbf{r}_{n, t}$, speed $\mathbf{v}_{n, t}$, acceleration $\dot{\mathbf{v}}_{n, t}$, and two clock errors ( $\delta_{n, t}$ for GPS and $\Delta_{n, t}$ for GLONASS) at time $t$ and evolves according to a matrix $\boldsymbol{\Phi}$, with a random variation $\mathbf{w}_{n, t-1}$, as follows:

$$
\mathbf{X}_{n, t}=\boldsymbol{\Phi} \mathbf{X}_{n, t-1}+\mathbf{w}_{n, t-1} \text {. }
$$

The measurement data $\mathbf{Y}_{n, t}=\left[\mathbf{p}_{n, t} ; \mathbf{v}_{n, t}\right]$ contains both pseudoranges $\mathbf{p}_{n, t}=\left[p_{n, t}^{(1)} ; p_{n, t}^{(2)} ; \ldots\right]$ and receiver speed $\mathbf{v}_{n, t}$ and is indirectly associated with the state $\mathbf{X}_{n, t}$ by the following equation $\left(\boldsymbol{\rho}_{n, t}=\left[\rho_{n, t}^{(1)} ; \rho_{n, t}^{(2)} ; \ldots\right]\right.$ is the true distance, $\mathbf{g}_{n, t}$ and $\mathbf{G}_{n, t}$ are flags indicating whether a satellite is GPS or GLONASS, and $\boldsymbol{\xi}_{n, t}$ is a measurement error):

$$
\mathbf{Y}_{n, t}=\left[\boldsymbol{\rho}_{n, t}+\mathbf{g}_{n, t} \cdot \delta_{n, t}+\mathbf{G}_{n, t} \cdot \Delta_{n, t} ; \mathbf{v}_{n, t}\right]+\boldsymbol{\xi}_{n, t} .
$$

$\mathbf{Y}_{n, t}$ can be linearized near a state $\mathbf{X}_{n, t}$, where a small change $\partial \mathbf{X}_{n, t}$ leads to a change $\mathbf{H}_{n, t} \cdot \partial \mathbf{X}_{n, t}$ in $\mathbf{Y}_{n, t}$.

With the variance matrix of $\mathbf{w}_{n, t-1}$ and $\boldsymbol{\xi}_{n, t}$ being $\mathbf{Q}$ and $\mathbf{R}$, respectively, the process of updating state $\mathbf{X}_{n, t}$ and its variance $\mathbf{P}_{n, t}$ for a receiver $n=a, b$ using pseudoranges of satellites in $\mathrm{S}$ is briefly described below as three main steps [17].

(1) Receiver state prediction (the predicted position of receiver $n, \mathbf{r}_{n, t}^{-}$, is obtained from $\left.\mathbf{X}_{n, t}^{-}\right)$:

$$
\mathbf{X}_{n, t}^{-}=\Phi \mathbf{X}_{n, t-1}^{+}, \mathbf{P}_{n, t}^{-}=\Phi \mathbf{P}_{n, t-1}^{+} \Phi^{T}+\mathbf{Q}
$$

(2) Pseudorange estimation: $p_{n, t}^{(s)-}$ is estimated from $\rho_{n, t}^{(s)}=$ $\left|\mathbf{r}_{n, t}^{-}-\mathbf{r}_{t}^{(s)}\right|$ by adding clock errors.
(3) Receiver state update: a Kalman gain $\mathbf{K}_{n, t}$ is computed based on the variance $\left(\mathbf{P}_{n, t}^{-}\right)$of predicted information and the variance $\mathbf{R}$ of measured information. Then, $\mathbf{X}_{n, t}^{-}$is updated to $\mathbf{X}_{n, t}^{+}$by adding the prediction error $\mathbf{Y}_{n, t}-\left(\mathbf{p}_{n, t}^{-} ; \mathbf{v}_{n, t}^{-}\right)$weighted by the Kalman gain using all satellites in $\mathbf{S}$, and $\mathbf{r}_{n, t}^{+}$is obtained from $\mathbf{X}_{n, t}^{+}$

$$
\begin{gathered}
\mathbf{K}_{n, t}=\mathbf{P}_{n, t}^{-} \cdot \mathbf{H}_{n, t}^{T} \cdot\left[\mathbf{H}_{n, t} \cdot \mathbf{P}_{n, t}^{-} \cdot \mathbf{H}_{n, t}^{T}+\mathbf{R}\right], \\
\mathbf{X}_{n, t}^{+}=\mathbf{X}_{n, t}^{-}+\mathbf{K}_{n, t} \cdot\left[\mathbf{Y}_{n, t}-\left(\mathbf{P}_{n, t}^{-} ; \mathbf{v}_{n, t}^{-}\right)\right], \\
\mathbf{P}_{n, t}^{+}=\left(\mathbf{I}-\mathbf{K}_{n, t} \mathbf{H}_{\mathrm{n}, t}\right) \mathbf{P}_{n, t}^{-} .
\end{gathered}
$$

The above Kalman filtering process is divided into two parts to accomplish both correlation detection of MPEs and position computation.

Correlation detection of MPEs involves three steps.

(i) For each common satellite in $\mathbf{S}$, if its elevation angle is above a threshold (e.g., $50^{\circ}$ ) or the SNR at both receivers is above a threshold (e.g., $44 \mathrm{~dB}$ ), it is added to a set $\mathbf{S}_{R}$; otherwise, it is added to a set $\mathbf{S}_{C}$. The thresholds are selected based on some initial analysis of the experiment data. Satellites in $\boldsymbol{S}_{R}$ are regarded as being reliable without MPEs and used in estimating current receiver positions, which are further used to detect correlation of MPEs of satellites in $\mathbf{S}_{\mathrm{C}}$.

(ii) Step 1-3 of Kalman filtering are performed for each receiver: the predicted position is $\mathbf{r}_{n, t}^{-}$in Step 1 and the updated position is $\mathbf{r}_{n, t}^{+}$using $\mathbf{S}_{R}$ instead of $\mathbf{S}$ in Step 3.

(iii) For each satellite $k \in S_{C}$, the metric in (3) is computed, using $\mathbf{r}_{n, t}^{+}$as the estimated receiver position. Satellites whose metrics are beyond a threshold are removed from $\mathbf{S}_{C}$. After the correlation detection, $\mathbf{S}_{C}$ becomes $\mathbf{S}_{C}^{\prime}$. MPEs of satellites in $\mathbf{S}_{C}^{\prime}$ are regarded as being spatially correlated.

In position computation, Step 3 of Kalman filtering is performed again, but using $\mathbf{S}_{R} \cup \mathbf{S}_{C}^{\prime}$ instead of $\mathbf{S}$. With the updated positions $\mathbf{r}_{a, t}^{+}$and $\mathbf{r}_{b, t}^{+}$, their difference is regarded as the relative position.

\section{Experiment Setup}

In the experiments, we used two types of receivers, $N$-receiver (NovAtel OEM6) and $U$-receiver ( $u$-blox EVK-6T), and two cars. As shown in Figure 2, two $N$-receivers $\left(N_{1}, N_{3}\right)$ and two $U$-receivers $\left(U_{1}, U_{3}\right)$ are installed on car 1 ; one $N$-receiver $\left(N_{2}\right)$ and one $U$-receiver $\left(U_{2}\right)$ are installed on car 2. Most of the time, two cars are driven along the same lane in a platoon, although they might temporarily change lanes, wait for signals at intersections, or be separated by other vehicles. In this way, we investigate the spatial correlation of MPEs at different interreceiver distances under practical vehicular scenarios.

In the experiment, car speed is obtained from the vehicle engine via the CAN-BUS (http://en.wikipedia.org/wiki/ CAN_bus), and a fibre optic gyroscope is used to estimate 


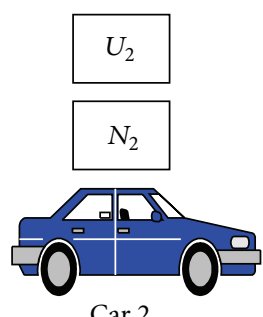

Car 2

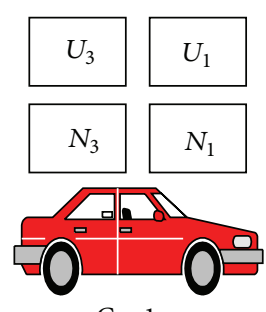

Car 1
FIGURE 2: Experiment setup: two $N$-receivers and two $U$-receivers on car 1 , one $N$-receiver and one $U$-receiver on car 2 . Two cars are driven in a platoon in urban areas with buildings on roadsides.

car's moving direction. From GNSS receivers, both pseudoranges and carrier phases are obtained and recorded. Receivers' positions, fixed by offline RTK-GPS processing with carrier phases [22], are used as ground truth. In the evaluation, pseudorange data of L1-code is used to compute relative positions between receivers.

\section{Statistics of Multipath Error}

In the analysis of MPE $m_{a}^{(k)}$ (in the pseudorange between receiver $a$ and satellite $k$ ), a nearby reference station is used as receiver $b$. As the reference station is set up in an open sky environment, its MPE of satellite $k, m_{b}^{(k)}$, is zero. Then, (3) is simplified as

$$
m_{a}^{(k)} \approx p_{a b}^{(k l)}-\rho_{a b}^{(k l)}
$$

by which $m_{a}^{(k)}$ can be estimated.

In the following, basic properties of MPE are first presented, and then, spatial correlation between MPEs at two receivers is analyzed.

4.1. Basic Properties of MPE. CDFs (cumulative distribution function [23]) of MPEs of two types of receivers are shown in Figure 3. For either receiver, MPEs in pseudoranges can be as large as $100 \mathrm{~m}$. The two types of receivers have different properties: $N$-receivers try to improve the precision of pseudoranges while $U$-receivers aim to improve the sensitivity. Accordingly, their MPEs also have different properties. Compared with $N$-receivers, $U$-receivers generally have larger MPEs. With a threshold $2.5 \mathrm{~m}, 78 \%$ MPEs of $N$ receivers are less than this threshold, while this probability decreases to 0.52 for $U$-receivers.

MPEs in pseudoranges are mainly caused by the reflection of positioning signals. In addition, a reflected signal, attenuated by each reflection, tends to have lower SNR (signalto-noise ratio). Therefore, there is some correlation between MPEs and SNR. CDFs of MPEs, under different SNRs, are shown in Figure 4. MPEs at high SNR (SNR $\geq 45)$ are very small. In comparison, MPEs at low SNR (SNR $\leq 40)$ may have large values.

The elevation angles of satellites affect the HDOP (horizontal dilution of precision), which is further associated with the positioning precision. In addition, the probability

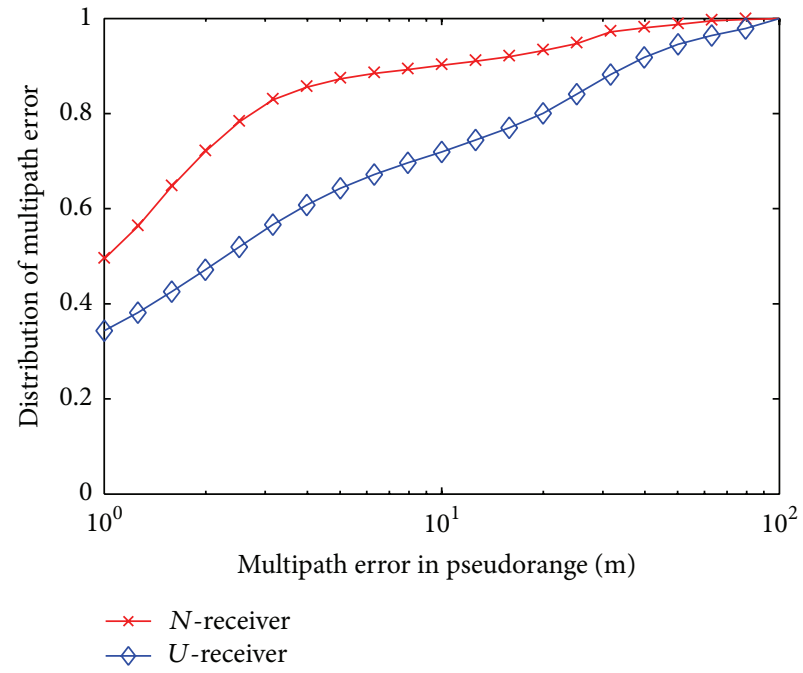

FIGURE 3: CDFs of MPEs in pseudoranges at two types of receivers.

that a positioning signal is obstructed and reflected increases when the elevation angle decreases. Therefore, MPEs are correlated with elevation angles of satellites. CDFs of MPEs, under different elevation angles, are shown in Figure 5. Generally, when elevation angle of a satellite is high (elevation angle $\geq 60$ ), its MPE is very small. Hence, a satellite with a high elevation angle can be used as a reference satellite in estimating MPEs of other satellites. MPEs get larger as elevation angles decrease. At low elevation angles, $U$-receivers tend to have larger MPEs than $N$-receivers. This is because $U$-receivers have higher sensitivity. Then, positioning signals with multiple reflections can be received as well, which contributes to large MPEs.

There is also some correlation between MPEs and moving speeds. Figure 6 shows CDFs of MPEs under different speeds. MPEs tend to have large values at low speeds. This can be explained as follows: GNSS receivers use tracking loop to track positioning signals; at low speeds, even a reflected positioning signal is relatively stable for some period, and it can enter the tracking loop of a receiver; at higher speeds, however, this becomes less possible.

4.2. Correlation between MPEs. Next, we investigate the correlation between MPEs at different interreceiver distances. Here, $N_{1}-N_{3}, U_{1}-U_{3}$ are installed on the same car and regarded as pairs of receivers with a short distance (about $1.5 \mathrm{~m}) . N_{1}-N_{2}, U_{1}-U_{2}$ are installed on different cars and regarded as pairs of receivers with a long distance, whose CDF is shown in Figure 7. The median distance is aound $10 \mathrm{~m}$ and the average distance is $19.4 \mathrm{~m}$.

In the following analysis, we focus on the satellites where either or both receivers have nonnegligible MPEs (MPE $\geq$ $5 \mathrm{~m}$ ). In other words, satellites that are directly visible to both receivers $(\mathrm{MPE} \approx 0)$ are not involved in the analysis.

As for MPEs of noncommon satellites, common satellites, and MPE difference of common satellites, their CDFs are 


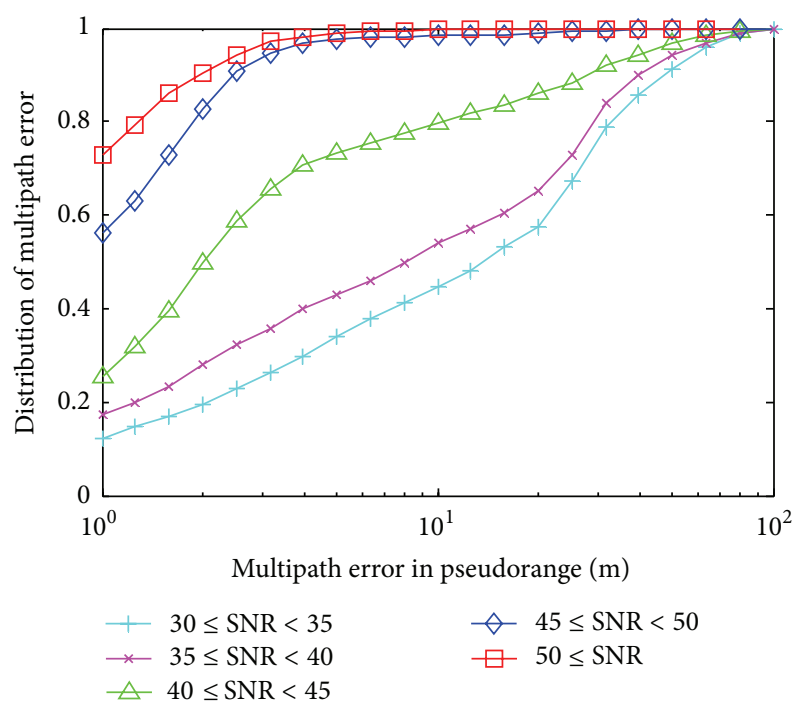

(a) $N$-receiver

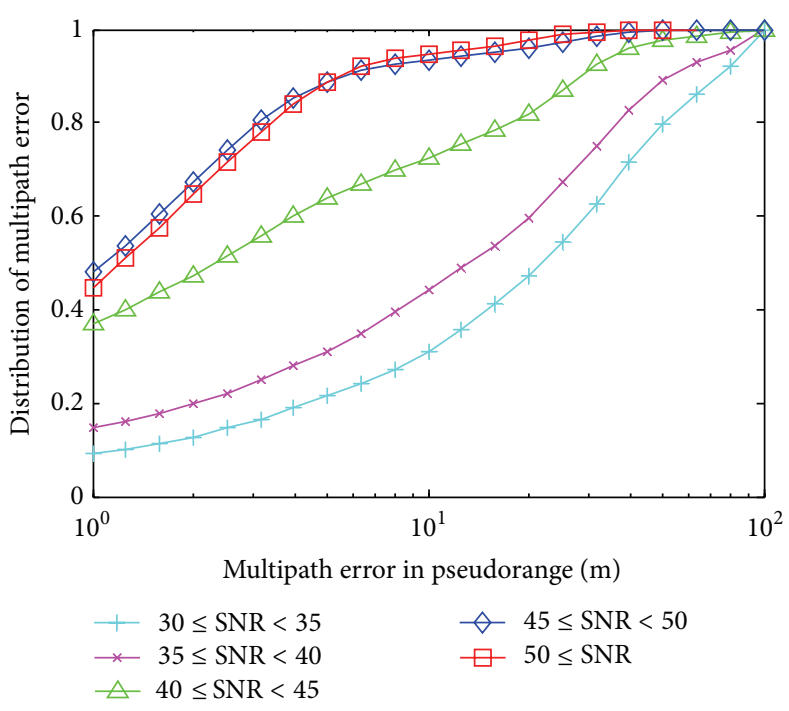

(b) $U$-receiver

FIGURE 4: CDFs of MPEs in pseudoranges under different SNR of received signals.

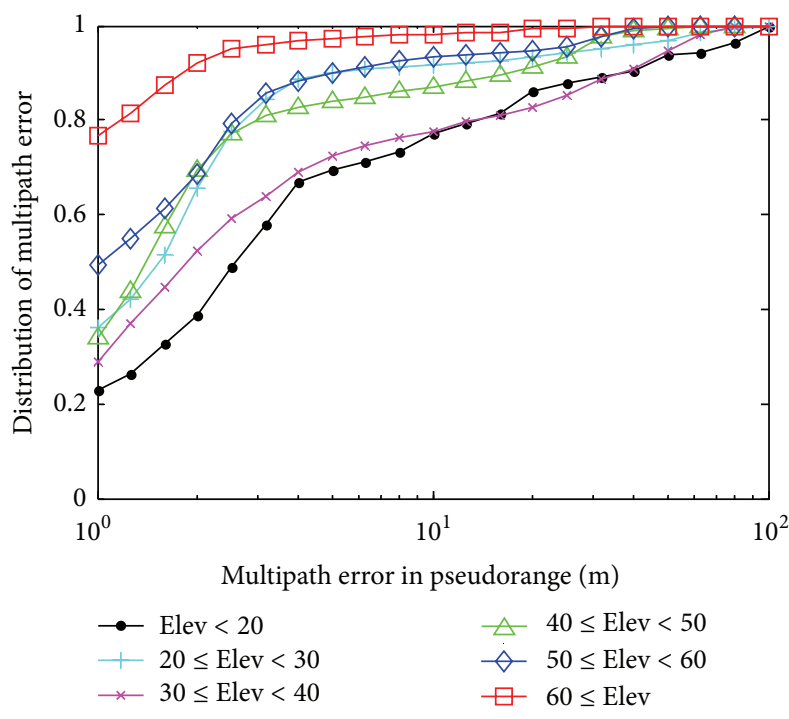

(a) N-receiver

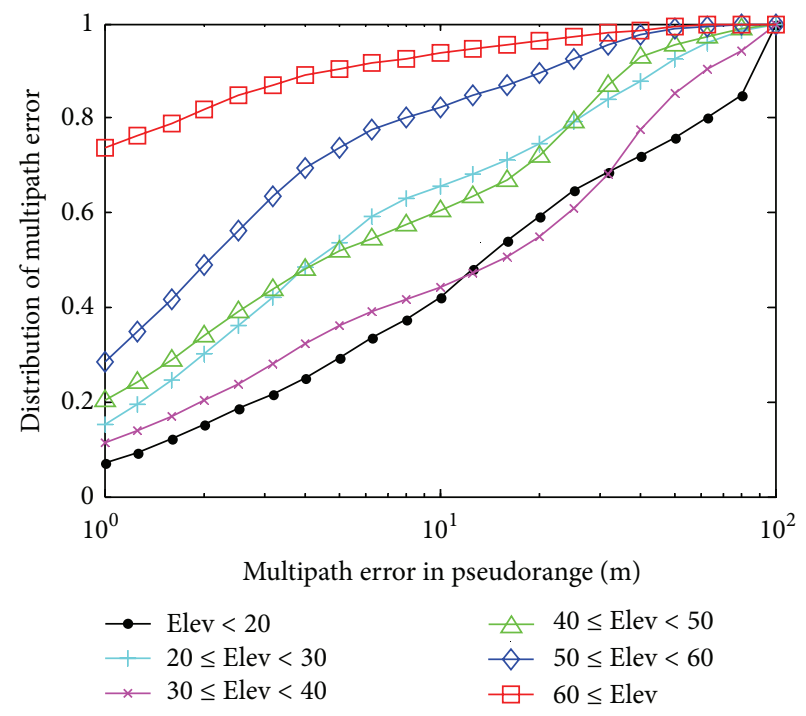

(b) $U$-receiver

FIGURE 5: CDFs of MPEs in pseudoranges under different elevation angles of satellites.

computed and shown in Figure 8. This figure reveals the following points.

(i) Under all cases, a MPE difference is much less than that of original MPEs. In other words, the concept of DGPS applies to two adjacent receivers since their MPEs can be cancelled by the differential operation.

(ii) The probability that a MPE difference is below a threshold (e.g., $10 \mathrm{~m}$ in Figure 8) is less than 1. Therefore, noncorrelated MPEs do exist, and some mechanisms are necessary to mitigate them.

(iii) As the interreceiver distance increases (e.g., (a) to (b) for $N$-receiver, (c) to (d) for $U$-receiver), the probability that a MPE difference is below a threshold (e.g., $10 \mathrm{~m}$ ) decreases. In other words, correlation between MPEs is inversely related with the interreceiver distance.

(iv) Compared with $N$-receivers, $U$-receivers tend to have lower correlation in MPEs (e.g., (a) to (c) where receivers are on the same car, (b) to (d) where receivers are on different cars).

(v) MPEs of common satellites are less than that of noncommon satellites. This is because a positioning signal is more likely a reflected one when it is only received by one receiver. 


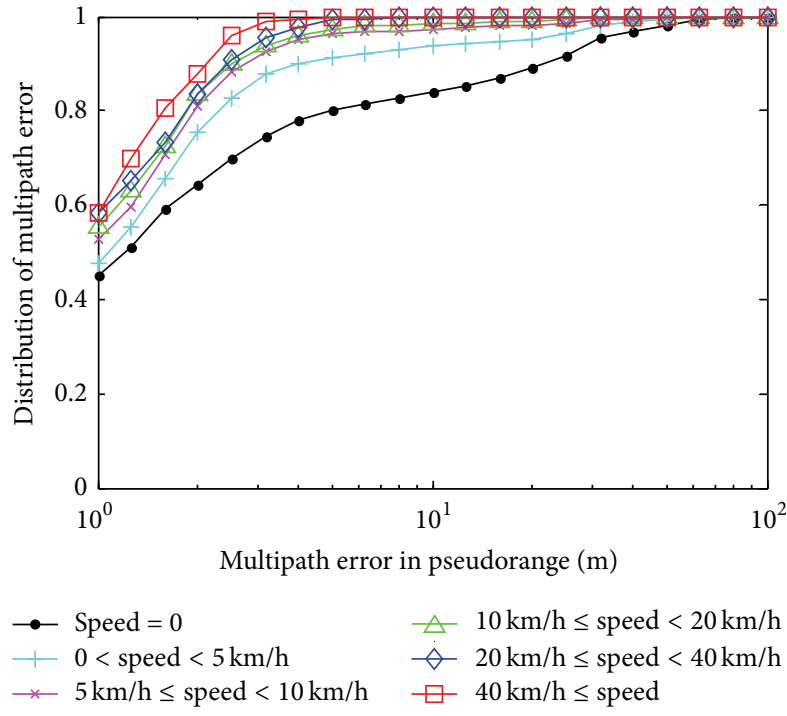

(a) $N$-receiver

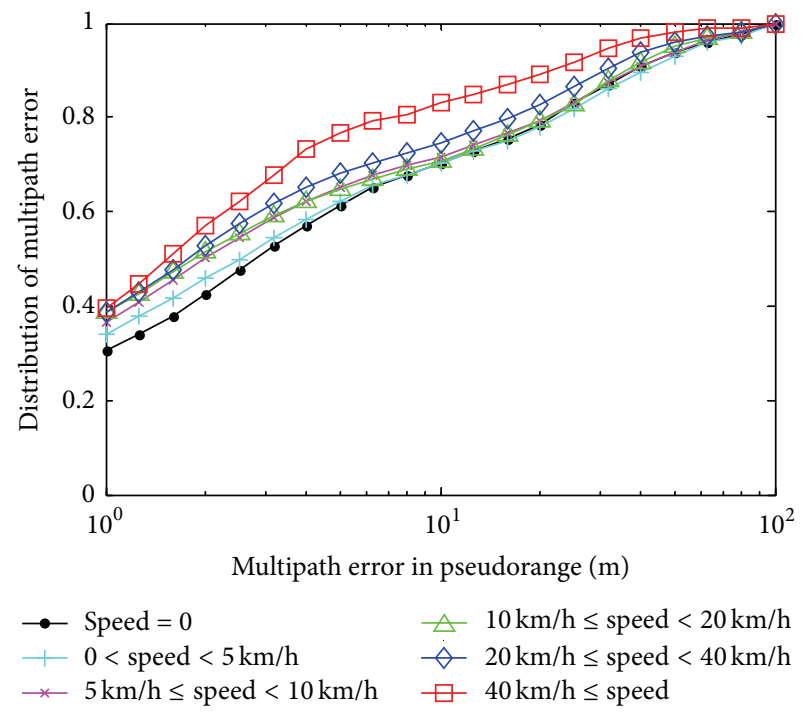

(b) $U$-receiver

FIGURE 6: CDFs of MPEs in pseudoranges under different moving speeds of receivers.

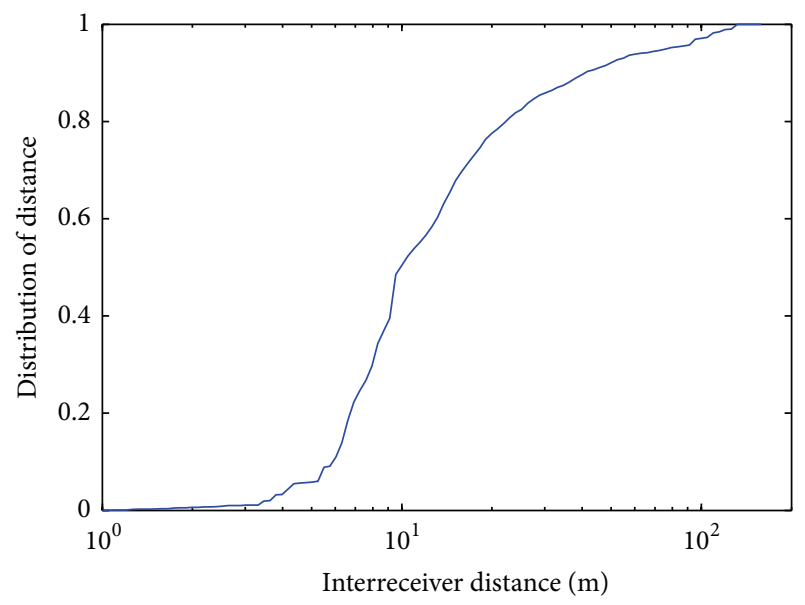

FIGURE 7: CDF of interreceiver distance when receivers are installed on top of separate cars.

The result in Figure 8 is summarized in Table 1. The probabilities that a MPE difference is below a certain threshold ( $5 \mathrm{~m}$ or $10 \mathrm{~m}$ ) are computed for different interreceiver distances (short distance, long distance) and different types of receivers ( $N$-receiver, $U$-receiver). With a relatively high probability, two receivers (especially $N$-receivers) have a correlation in their MPEs, although this correlation decreases at longer distances.

We further study the correlation between MPEs by investigating the distribution of MPE pairs (two MPEs at two receivers). Their normalized histograms are shown in Figure 9. In Figures 9(a) and 9(c), two receivers are installed in the same car with a short distance, and most MPE pairs lie along the diagonal of the horizontal axis. As the interreceiver distance increases (from (a) to (b) or from (c) to (d)), the
TABLE 1: Probability of MPEs under different scenarios.

\begin{tabular}{lcc}
\hline & $N$-receiver & $U$-receiver \\
\hline Prob(MPE diff <5), short distance & 0.537 & 0.329 \\
Prob(MPE diff <5), long distance & 0.364 & 0.203 \\
Prob(MPE diff <10), short distance & 0.743 & 0.607 \\
Prob(MPE diff <10), long distance & 0.579 & 0.440 \\
Prob(single MPE), short distance & 0.093 & 0.126 \\
Prob(single MPE), long distance & 0.184 & 0.234 \\
\hline
\end{tabular}

peaks of histogram decrease and points are spread in a wider area. Even in this case, there is still a high probability that MPE pairs are near the diagonal, which confirm the correlation between MPEs, though the probability is less compared with that at a shorter distance. Again, MPEs of $N$ receivers tend to have higher correlation than those of $U$ receivers.

There are some points on either axis, which indicates that MPE is only found at one receiver, but with a negligible value at the other receiver. This corresponds to the case of car $B$ and car $C$ in Figure 1. In this case, the MPEs have low correlation, and the associated common satellite should be removed before fixing relative positions. The probabilities associated with this case are shown in the last two rows of Table 1 .

As discussed before, the distribution of MPEs depends on moving speeds of receivers. A natural question is whether the correlation in MPEs is affected by speeds as well. We computed MPE difference of two receivers, when both speeds are less than $0.1 \mathrm{~km} / \mathrm{h}$ or both are greater than $10 \mathrm{~km} / \mathrm{h}$ (we did so because two receivers have different speeds in the long distance case). The CDFs of MPE of $N$ receivers are shown in Figure 10, which shows a clear distinction between the MPE distributions under low and high speeds. 


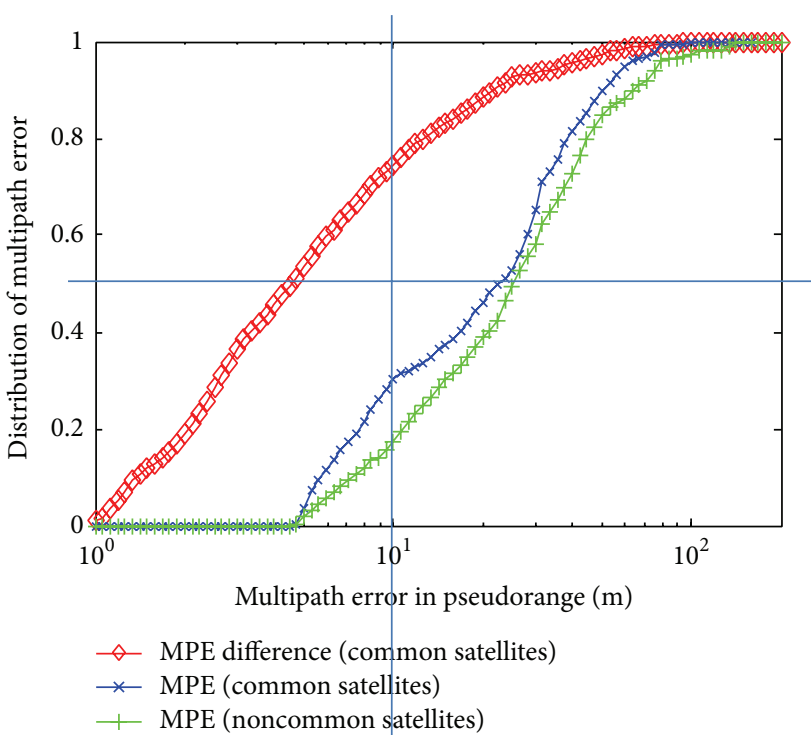

(a) $\mathrm{N}$-receiver, short distance

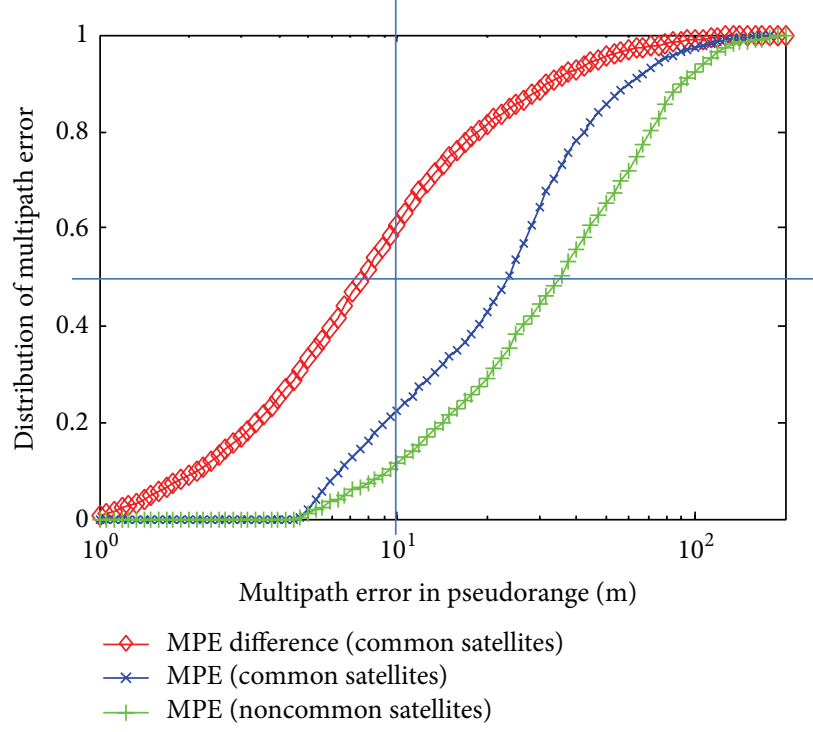

(c) $U$-receiver, short distance

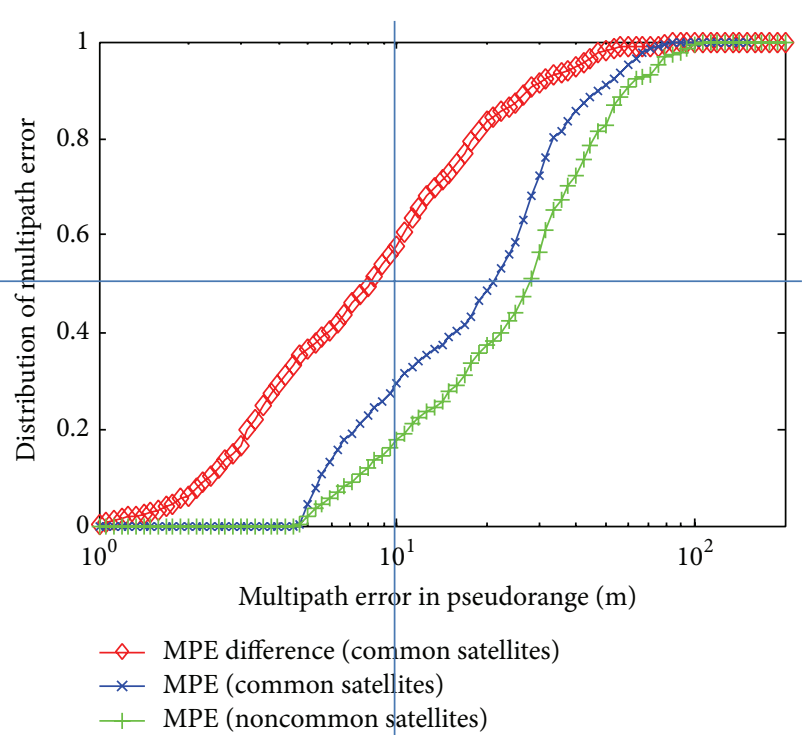

(b) $N$-receiver, long distance

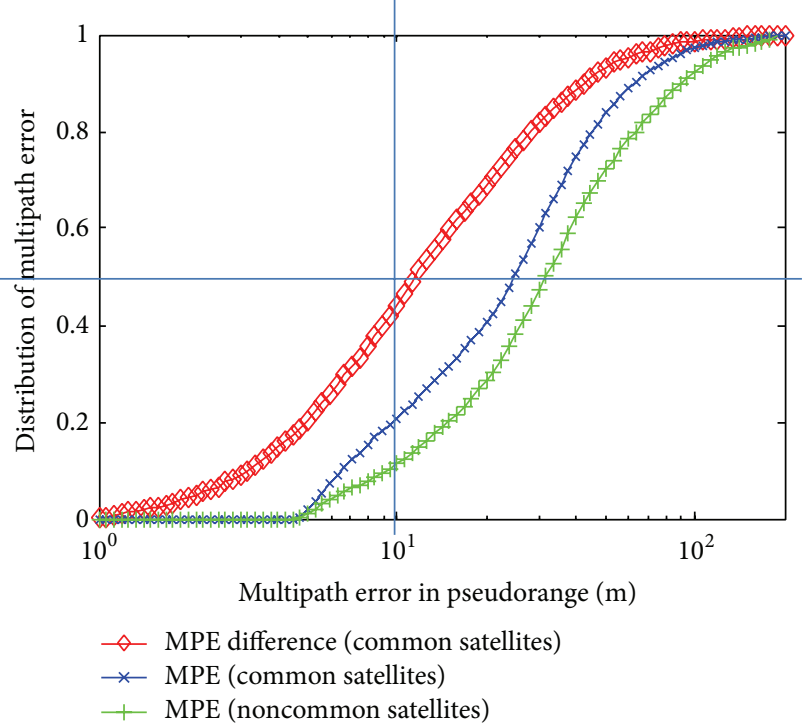

(d) $U$-receiver, long distance

FIGURE 8: CDFs of MPEs of noncommon satellites, common satellites, and MPE difference of common satellites. (a) Two N-receivers with a short distance, (b) two $N$-receivers with a long distance, (c) two $U$-receivers with a short distance, and (d) two $U$-receivers with a long distance.

\section{Refining the CoRelPos Scheme}

Here, we further refine the CoRelPos scheme by considering the dynamics of MPEs.

A Kalman filter is used to combine the predicted position (based on previous position and speed) and measured pseudoranges to estimate the receiver position. Both predicted position and pseudoranges have a variance indicating their potential errors. From these variances, a Kalman gain is computed, and the new position is computed as the weighted sum of predicted position and the prediction error. The precision of predicted position depends on the accuracy of moving speed. It will have a larger weight when the prediction variance (indirectly, the speed variance) is small; on the other hand, the prediction error (pseudoranges) should have a large weight when the pseudoranges have small variances.

Typically, fixed variances are assigned for pseudoranges and moving speeds. But in the above analysis, we find that MPEs are related with speeds, besides elevation angles of satellites. Therefore, the variances of pseudoranges and speeds are adjusted as follows.

(i) A small variance for pseudoranges at a high speed and a large variance at a low speed: in other words, reflected positioning signals will be more leveraged at higher speeds. This is based on the relationship between MPE difference and speeds shown in Figure 10. 


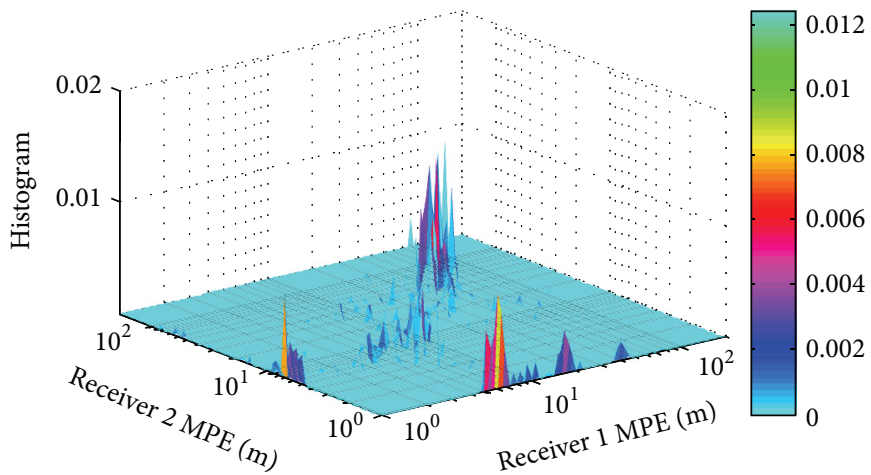

(a) $N$-receiver, short distance

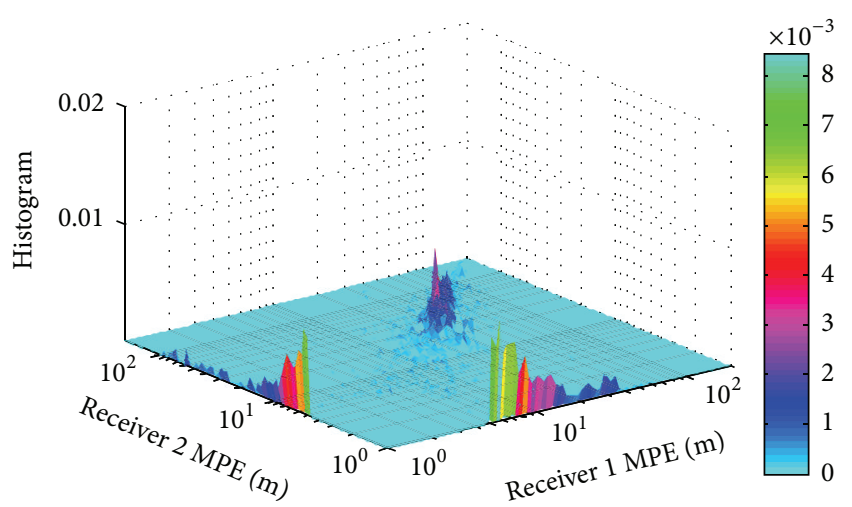

(c) U-receiver, short distance

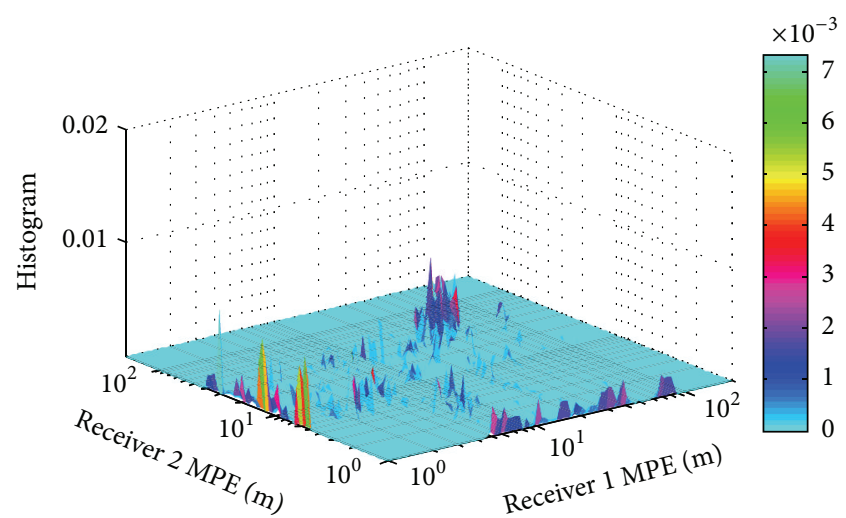

(b) $N$-receiver, long distance

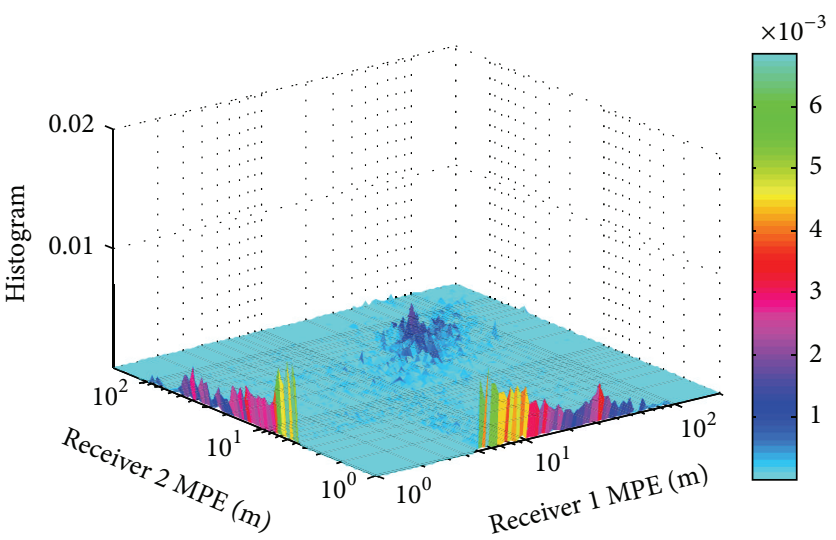

(d) $U$-receiver, long distance

FIgURE 9: Histograms of MPE pairs. (a) Two $N$-receivers with a short distance, (b) two $N$-receivers with a long distance, (c) two $U$-receivers with a short distance, and (d) two $U$-receivers with a long distance.

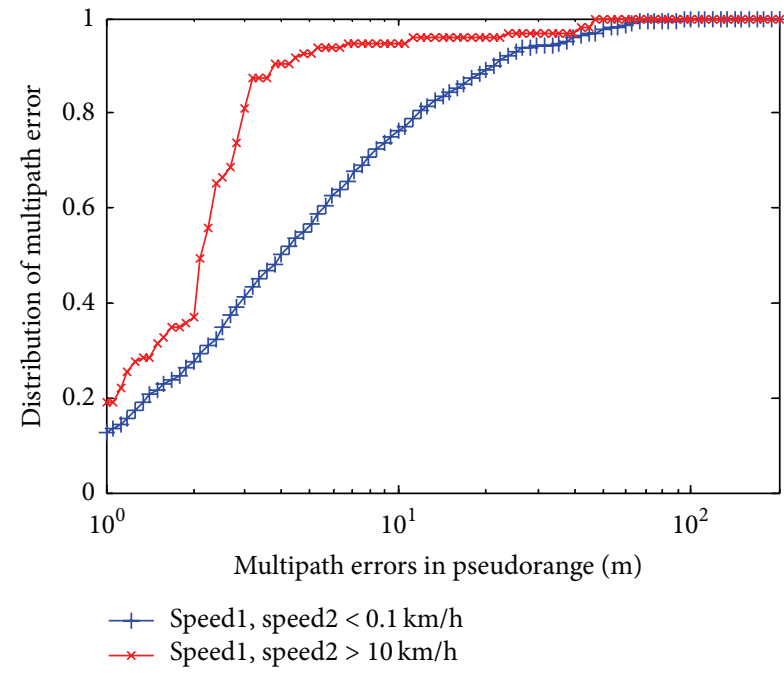

(a) $N$-receiver, short distance

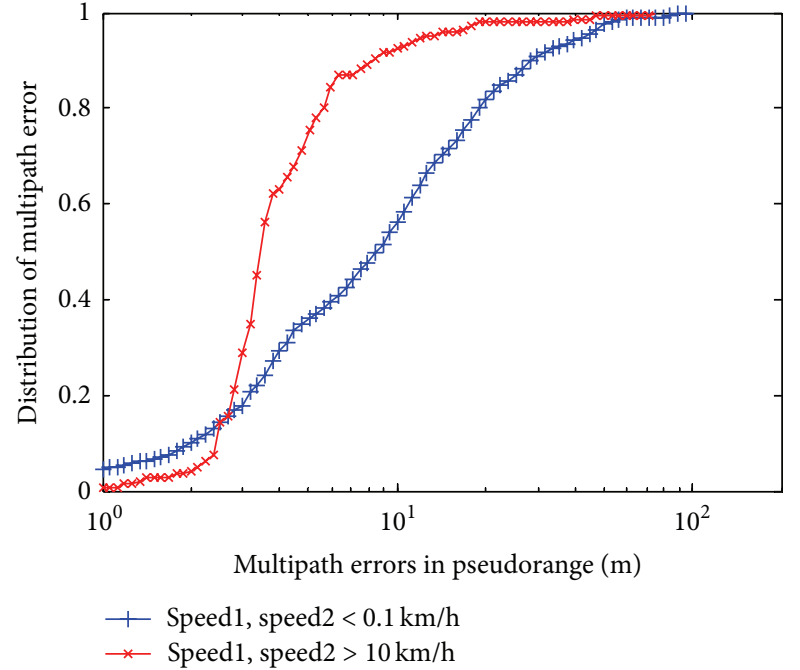

(b) $N$-receiver, long distance

FIGURE 10: CDFs of MPE difference under different moving speeds. (a) Two $N$-receivers with a short distance and (b) two $N$-receivers with a long distance. 
(ii) A large variance for speed when the moving direction changes, a small variance otherwise, and 0 variance when the receiver stops (when speed pulse gets equal to 0 ): in our analysis, we found that the estimation error of speed tends to be higher when the moving direction changes. In addition, when a receiver (car) comes to a stop, reflected positioning signals not received in the moving period start to be received, but with large noncorrelated MPEs. In such cases, it is more reliable to trust the speed (with 0 variance).

This refined CoRelPos scheme is called CoRelPos+.

As analyzed in [20], there is a high correlation in MPEs when two vehicles run on the same road and share the same reflective surface. In this case, reflected signals, being correlated, can be leveraged in relative positioning. On the other hand, when two vehicles run on different roads and have different reflective surfaces, the correlation in their MPEs will be low. Then, most reflected signals, being noncorrelated, will be removed, although the shortage of satellites remains.

In conventional positioning schemes, a vehicle computes its own absolute position independent of other vehicles, and the computation time per epoch, $T_{\mathrm{CONV}}=T_{1}+T_{2}$, is mainly composed of two parts, $T_{1}$ for computing satellite positions and $T_{2}$ for computing the vehicle position. In CoRelPos(+), a vehicle computes the relative position of each of its $N$ adjacent neighbors, but the satellite positions are computed only once. For each neighbor, predicting the position per vehicle via Kalman filtering takes a time $T_{3}$ and the total time for a pair of vehicles is $2 T_{3}$; the correlation detection in MPEs takes a time $T_{4}$, and finally computing the pair of positions again using correlated signals roughly takes a time $2 T_{3}$. Altogether, the computation cost per pair of vehicles is $T_{\text {CoRelPos }}=T_{1}+4 T_{3}+T_{4}$, where $T_{4}$ is relatively small compared with $T_{3}$ and can be neglected. Then, the total computation cost per vehicle per epoch will be $T_{1}+N \cdot 4 T_{3}$, linearly increasing with $N$. In the prototype testbed we built using the free software RTKLIB [22], the parameters $T_{1}$ and $T_{\mathrm{CONV}}$ are equal to $0.34 \mathrm{~ms}$ and $0.73 \mathrm{~ms}$, respectively, and $T_{\text {CoRelPos }}$ is equal to $1.51 \mathrm{~ms}$. Accordingly, the computation time of CoRelPos(+) under $N$ neighbors is equal to $0.39+1.12 \mathrm{~N}$ ms.

The precision of relative positions only gets important when the intervehicle distance gets short. This can be used to reduce the computation cost and $\operatorname{CoRelPos}(+)$ works as follows: each vehicle always performs conventional positioning schemes and gets a rough estimation of distances to nearby vehicles; when the distance to an adjacent vehicle gets less than a threshold (e.g., less than $50 \mathrm{~m}$ ), an instance of CoRelPos $(+)$ is started for accurate relative positioning; in this way, each vehicle only needs to compute the relative positions for a small number (a small $N$ ) of its adjacent vehicles. Further reduction of the computation cost is left as a future work.

5.1. A Short Comparison. In the experiment, we will evaluate five schemes, as follows.

(i) N-receiver: the relative position is directly computed from the positions output by $N$-receivers. There is no
TABLE 2: Comparison of different schemes.

\begin{tabular}{lcccc}
\hline & Comm. & KF & Corr. & Dyna. \\
\hline CommSat & Yes & No & No & No \\
KF + CommSat & Yes & Yes & No & No \\
CoRelPos & Yes & Yes & Yes & No \\
CoRelPos+ & Yes & Yes & Yes & Yes \\
\hline
\end{tabular}

coordination between receivers when deciding which satellites to use in fixing positions.

(ii) CommSat: only satellites commonly available to both receivers are used, the same as in DGPS.

(iii) $\mathrm{KF}+\mathrm{CommSat}$ : based on CommSat, a Kalman filter (speed information) is used to smooth the positions. This represents an implementation of state-of-the-art positioning methods.

(iv) CoRelPos [20]: based on $\mathrm{KF}+\mathrm{CommSat}$, pseudoranges with noncorrelated MPEs are removed before fixing positions.

(v) CoRelPos+: the refined CoRelPos scheme.

A comparison of these schemes is listed in Table 2. These schemes implement part or all of the four features, Comm. (using common satellites), KF (using a Kalman filter), Corr. (conducting correlation detection of MPEs), and Dyna. (considering MPE dynamics).

\section{Results of Positioning}

The evaluation of CoRelPos was conducted in [20] by setting two receivers on stationary cars or on one car with a short interreceiver distance. In this work, we installed two receivers on two separate cars with a longer interreceiver distance, aiming to make the experiment scenario as practical as possible. With the collected data of pseudoranges and car speeds, we fixed the relative positions offline and computed the distribution of horizontal positioning errors by comparing with the ground truth. As $U$-receivers have quite complex properties in their MPEs, in the following, we mainly use the data of $N$-receivers, which have shown higher correlation in MPEs.

Three scenarios and their relative positioning errors are shown in Figures 11, 12, and 13, respectively, where the experiment was done in January 2014. In all three scenarios, CoRelPos can effectively reduce the positioning errors compared with other schemes. The positioning precision is further improved by CoRelPos+. The positioning error is especially small in scenario 3, which can be explained as follows: in scenarios 1 and 2, the cars are driven in an area (Yaesu near Tokyo station) with high buildings on the roadsides; the number of directly visible satellites is small; in addition, there are frequent changes in the moving direction of receivers; in scenario 3 cars are driven from Yaesu to the Etchujima Island, and there are fewer tall buildings on roadsides and fewer changes in moving directions.

Positioning precision depends on the constellation of satellites, which is time-variant. Figure 14 shows the CDFs of 

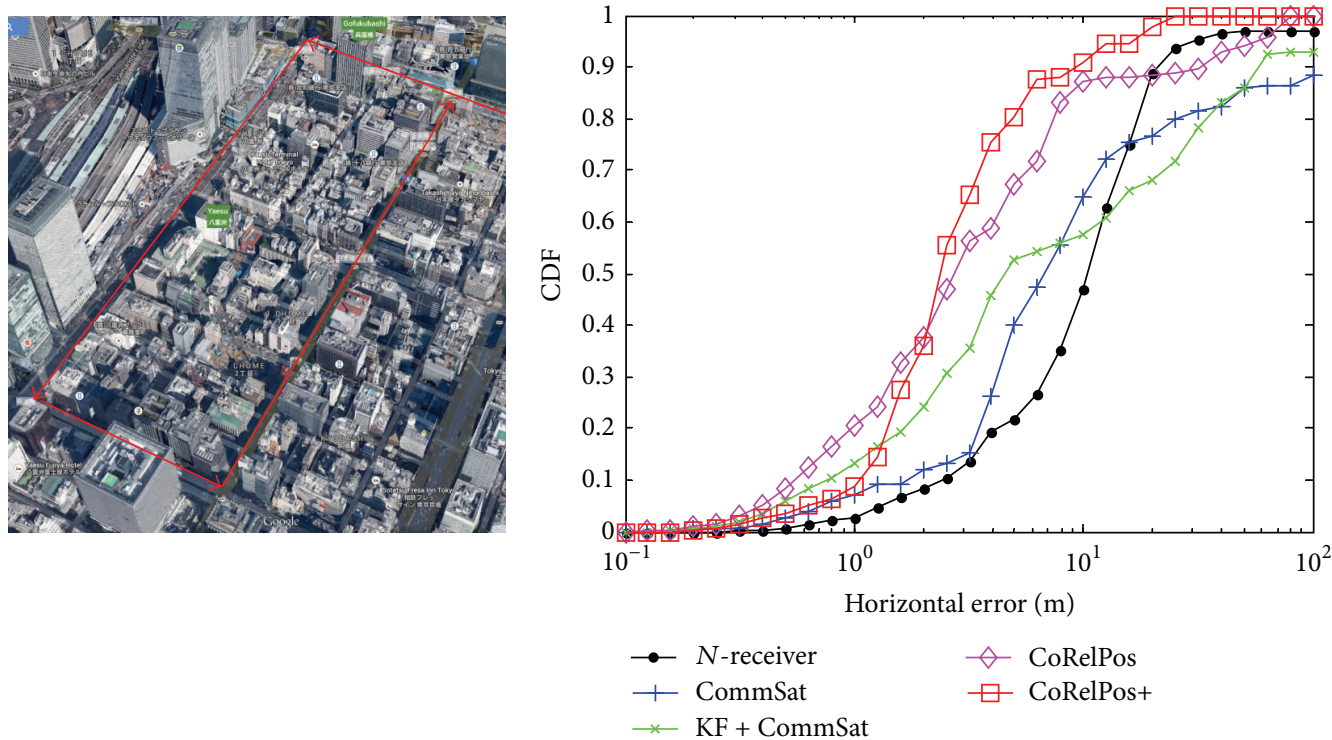

FIGURE 11: Scenario 1. CDFs of horizontal, relative positioning errors by different schemes.
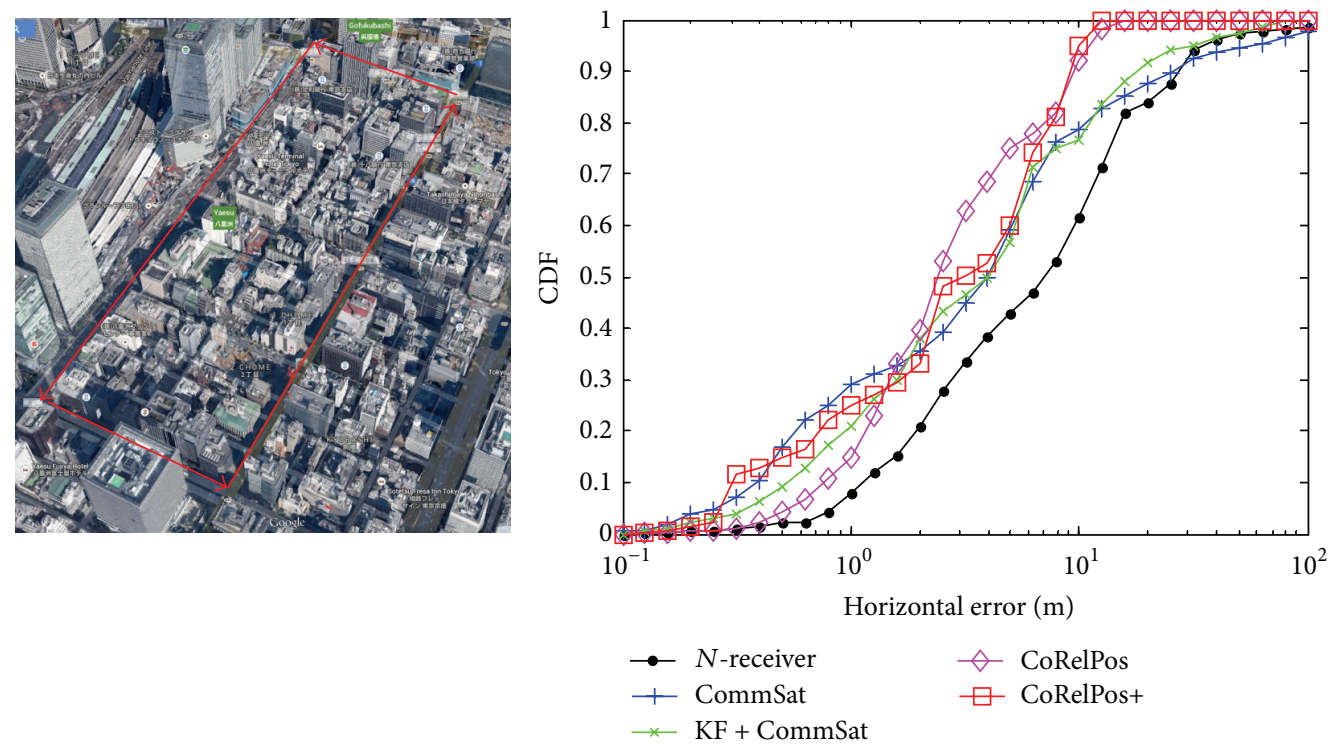

FIGURE 12: Scenario 2. CDFs of horizontal, relative positioning errors by different schemes.

horizontal, relative positioning errors around Yaesu, but on a different day in October 2013. This reflects a similar trend as in Figures 11, 12, and 13.

The RMS (root mean square) errors of five schemes, under four scenarios, are further summarized in Table 3. Compared with directly using $N$-receivers, coordinating receivers to use common satellites usually can reduce the errors. Exceptions do exist (e.g., scenario 1) because fewer satellites are available in the latter case. This is also confirmed by Table 4, which indicates that CommSat usually has a low rate of fixing positions. Combining position prediction with satellite positioning via $\mathrm{KF}+\mathrm{CommSat}$ can reduce the outage and further reduce the errors, but its effect is limited by the MPEs. Removing noncorrelated MPEs, CoRelPos can greatly
TABLE 3: RMS errors of horizontal, relative positioning errors by different schemes (unit: $\mathrm{m}$ ).

\begin{tabular}{lcccc}
\hline & Scen. 1 & Scen. 2 & Scen. 3 & Scen. 4 \\
\hline$N$-receiver & 53.03 & 32.96 & 35.59 & 29.33 \\
CommSat & 157.63 & 29.34 & 18.71 & 14.81 \\
KF + CommSat & 49.03 & 17.61 & 12.19 & 9.57 \\
CoRelPos & 22.76 & 6.44 & 5.44 & 5.42 \\
CoRelPos+ & 7.63 & 6.40 & 1.54 & 3.48 \\
\hline
\end{tabular}

reduce the errors. These errors can be further reduced by considering the dynamics of MPE correlations in CoRelPos+. 

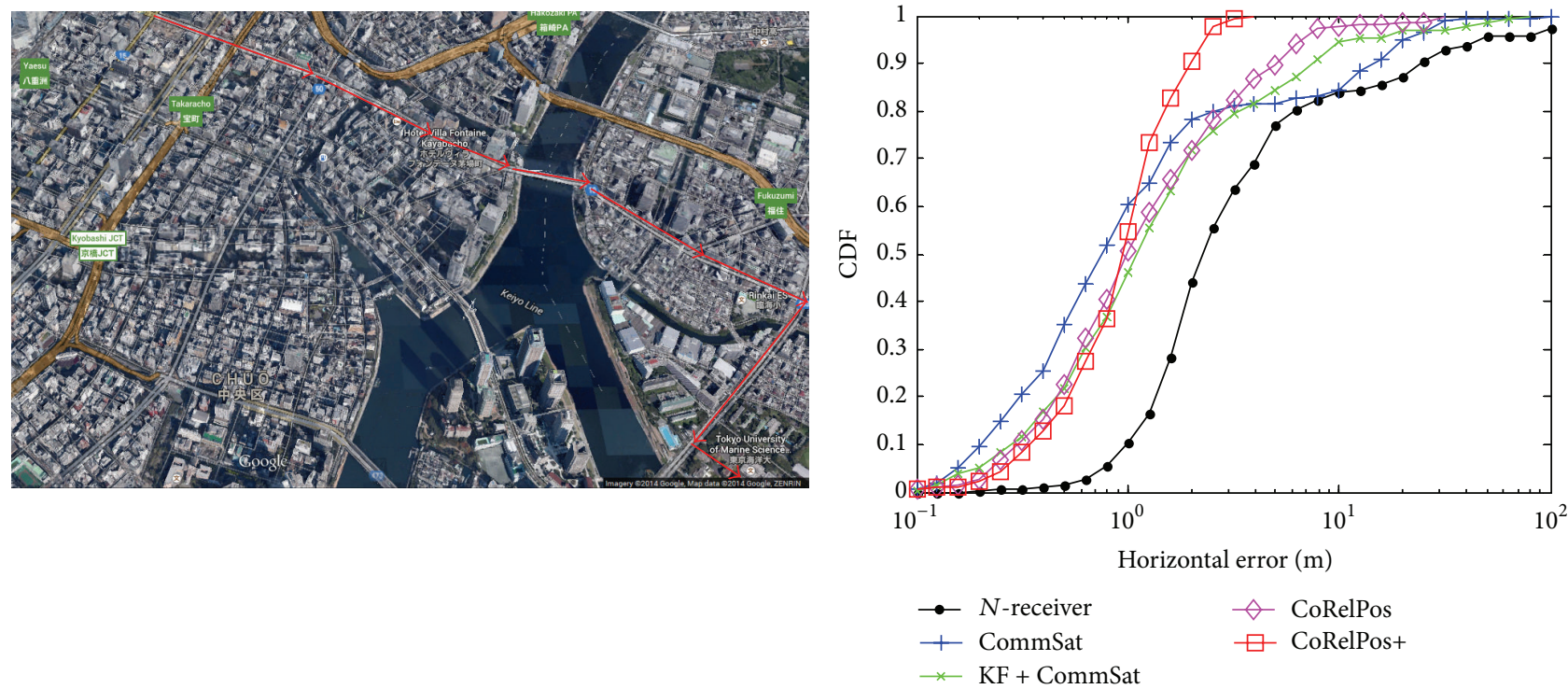

FIGURE 13: Scenario 3. CDFs of horizontal, relative positioning errors by different schemes.
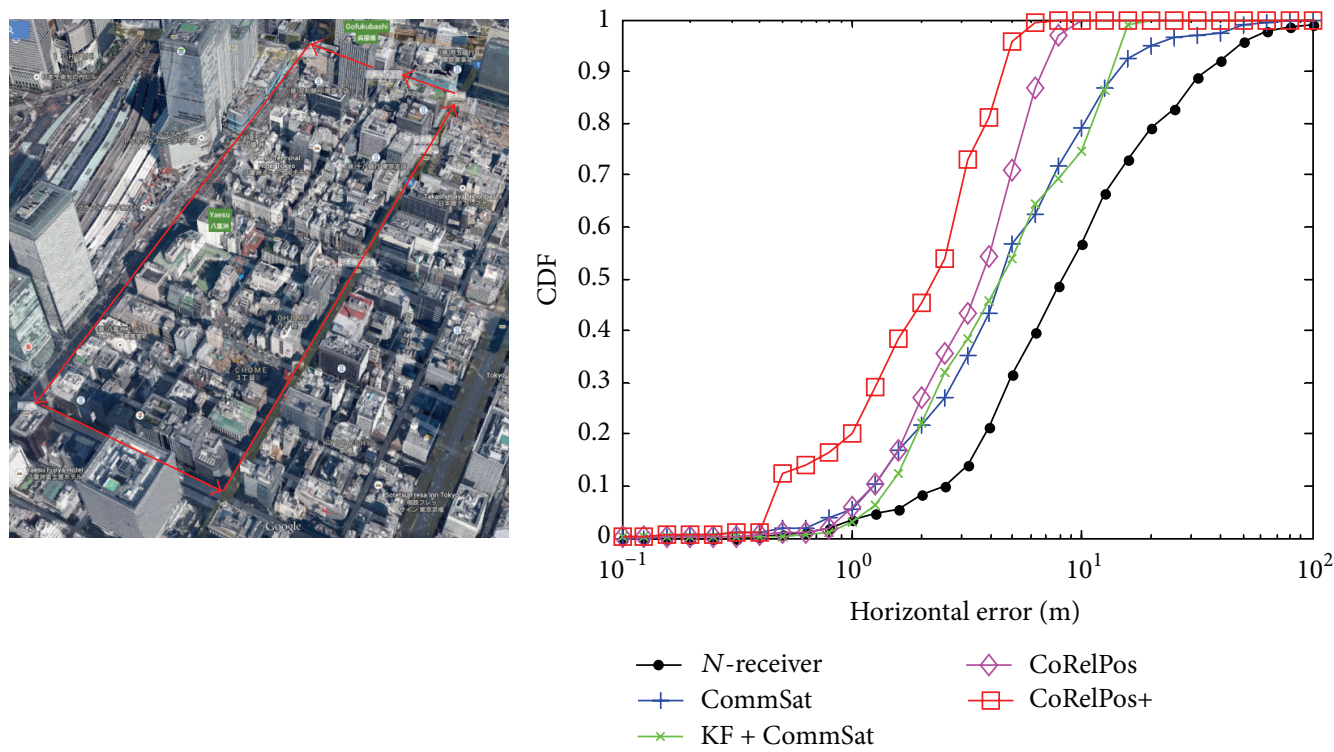

FIGURE 14: Scenario 4. CDFs of horizontal, relative positioning errors by different schemes.

TABLE 4: Percentage of epochs that relative positions can be fixed.

\begin{tabular}{lcccc}
\hline & Scen. 1 & Scen. 2 & Scen. 3 & Scen. 4 \\
\hline$N$-receiver & $85.7 \%$ & $68.5 \%$ & $63.4 \%$ & $75.6 \%$ \\
CommSat & $82.9 \%$ & $42.6 \%$ & $24.8 \%$ & $63.3 \%$ \\
KF + CommSat & $100 \%$ & $99.1 \%$ & $100 \%$ & $95.3 \%$ \\
CoRelPos & $100 \%$ & $99.1 \%$ & $100 \%$ & $95.3 \%$ \\
CoRelPos+ & $100 \%$ & $99.1 \%$ & $100 \%$ & $95.3 \%$ \\
\hline
\end{tabular}

\section{Conclusion}

Precision positioning in urban canyons remains a challenge due to the obstructions and reflections of roadside buildings.
In this paper, analysis of experiment data reveals different properties of MPEs, especially their spatial correlation in local areas and the dependency of MPE correlations on moving speeds. Considering the dynamics of MPE helps further improve positioning precision. The evaluation of relative positioning by using two receivers on two cars makes the proposed scheme one step towards practical applications. In the future, we will continue the studies of the $U$-receivers which have more complex properties of MPEs.

\section{Conflict of Interests}

The authors declare that there is no conflict of interests regarding the publication of this paper. 


\section{Acknowledgment}

This work is supported by the Strategic Information and Communications R\&D Promotion Programme (SCOPE) funded by Ministry of Internal Affairs and Communications, Japan.

\section{References}

[1] P. Misra and P. Enge, Global Positioning System: Signals, Measurements, and Performance, Ganga-Jamuna, 2nd edition, 2006.

[2] M. L. Sichitiu and M. Kihl, "Inter-vehicle communication systems: a survey," IEEE Communications Surveys and Tutorials, vol. 10, no. 2, pp. 88-105, 2008.

[3] ASV4, http://www.soumu.go.jp/main_sosiki/joho_tsusin/policyreports/chousa/its/pdf/ 081219_2_si2-4.pdf.

[4] O. Shagdar, S. Tang, M. N. Shirazi, R. Suzuki, and S. Obana, "Reliable cut-through forwarding in CDMA inter-vehicle networks," in Proceedings of the 2nd International Conference on Ubiquitous Information Management and Communication (ICUIMC '08), pp. 491-495, February 2008.

[5] A. J. Van Dierendonck, P. Fenton, and T. Ford, "Theory and performance of narrow correlator spacing in a GPS receiver," Navigation, Journal of the Institute of Navigation, vol. 39, no. 3, pp. 265-283, 1992.

[6] B. Townsend and P. Fenton, "A practical approach to the reduction of pseudorange multipath errors in a L1 GPS receiver," in Proceedings of the 7th International Technical Meeting of the Satellite Division of the Institute of Navigation, pp. 143-148, September 1994.

[7] V. A. Veitsel, A. V. Zhdanov, and M. I. Zhodzishsky, “The mitigation of multipath errors by strobe correlators in GPS/GLONASS receivers," GPS Solutions, vol. 2, no. 2, pp. 38-45, 1998.

[8] J. W. Betz, "Binary offset carrier modulations for radionavigation," Navigation, Journal of the Institute of Navigation, vol. 48, no. 4, pp. 227-246, 2001.

[9] R. Hatch, "The synergism of GPS code and carrier measurements," in Proceedings of the 3rd International Geodetic Symposium on Satellite Doppler Positioning, pp. 1213-1231, 1983.

[10] R. D. J. van Nee, J. Siereveld, P. C. Fenton, and B. R. Townsend, "Multipath estimating delay lock loop: approaching theoretical accuracy limits," in Proceedings of the IEEE Position Location and Navigation Symposium, pp. 246-251, Las Vegas, Nev, USA, April 1994.

[11] S. Rougerie, G. Carrié, F. Vincent, L. Ries, and M. Monnerat, "A new multipath mitigation method for GNSS receivers based on an antenna array," International Journal of Navigation and Observation, vol. 2012, Article ID 804732, 11 pages, 2012.

[12] Y. Suh and R. Shibasaki, "Evaluation of satellite-based navigation services in complex urban environments using a threedimensional GIS," IEICE Transactions on Communications, vol. 90, no. 7, pp. 1816-1825, 2007.

[13] J.-I. Meguro, T. Murata, J.-I. Takiguchi, Y. Amano, and T. Hashizume, "GPS multipath mitigation for urban area using omnidirectional infrared camera," IEEE Transactions on Intelligent Transportation Systems, vol. 10, no. 1, pp. 22-30, 2009.

[14] M. Z. Win, A. Conti, S. Mazuelas et al., "Network localization and navigation via cooperation," IEEE Communications Magazine, vol. 49, no. 5, pp. 56-62, 2011.

[15] IEEE, "Wireless LAN medium access protocol (MAC) and physical layer (PHY) specification. Ammendment 6: wireless access in vehicular environment," IEEE Std 802.11p, 2010.
[16] N. Alam, A. T. Balaei, and A. G. Dempster, "Positioning enhancement with double differencing and DSRC", in Proceedings of the 23rd International Technical Meeting of the Satellite Division of the Institute of Navigation (ION GNSS '10), pp. 12101218, September 2010.

[17] P. D. Groves, Principles of GNSS, Inertial, and Multi Sensor Integrated Navigation Systems, Artech House, 2007.

[18] A. Taha, C. Hancock, G. Roberts, and X. Meng, "The use of GPS and INS for centimeter precision during large GPS outages," in Proceedings of the International Symposium on GPS/GNSS, 2008.

[19] E. D. Martí, D. Martín, J. García, A. de la Escalera, J. M. Molina, and J. M. Armingol, "Context-aided sensor fusion for enhanced urban navigation," Sensors, vol. 12, no. 12, pp. 16802-16837, 2012.

[20] S. Tang, N. Kubo, N. Kawanishi, R. Furukawa, A. Hasegawa, and Y. Takeuchi, "Cooperative relative positioning for intelligent transportation system," International Journal of Intelligent Transportation Systems Research, 2014.

[21] H. Musoff and P. Zarchan, Fundamentals of Kalman Filtering: A Practical Approach, American Institute of Aeronautics and Astronautics, 2nd edition, 2005.

[22] T. Takasu, N. Kubo, and A. Yasuda, "Development, evaluation and application of RTKLIB: a program library for RTK-GPS," in Proceedings of the International Symposium on GPS/GNSS, November 2007.

[23] D. P. Bertsekas and J. N. Tsitsiklis, Introduction to Probability, Athena Scientific, Belmont, Mass, USA, 2002. 

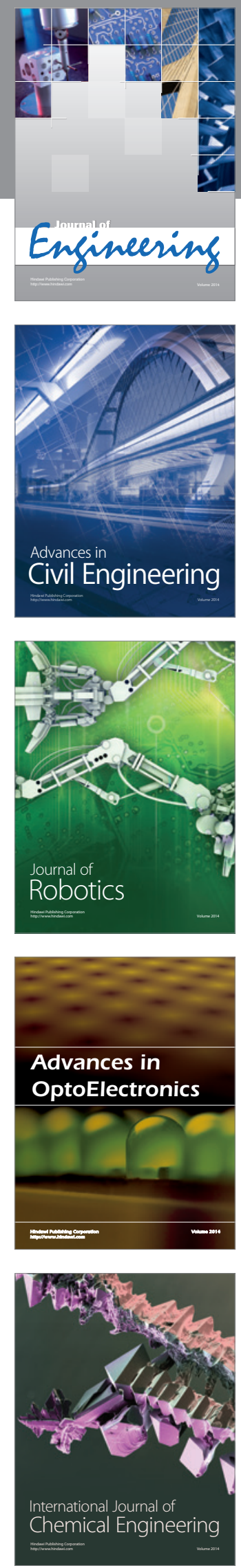

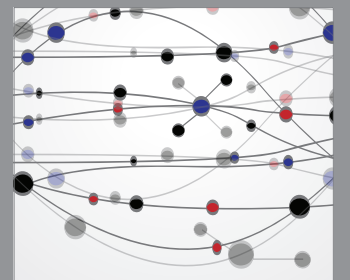

The Scientific World Journal
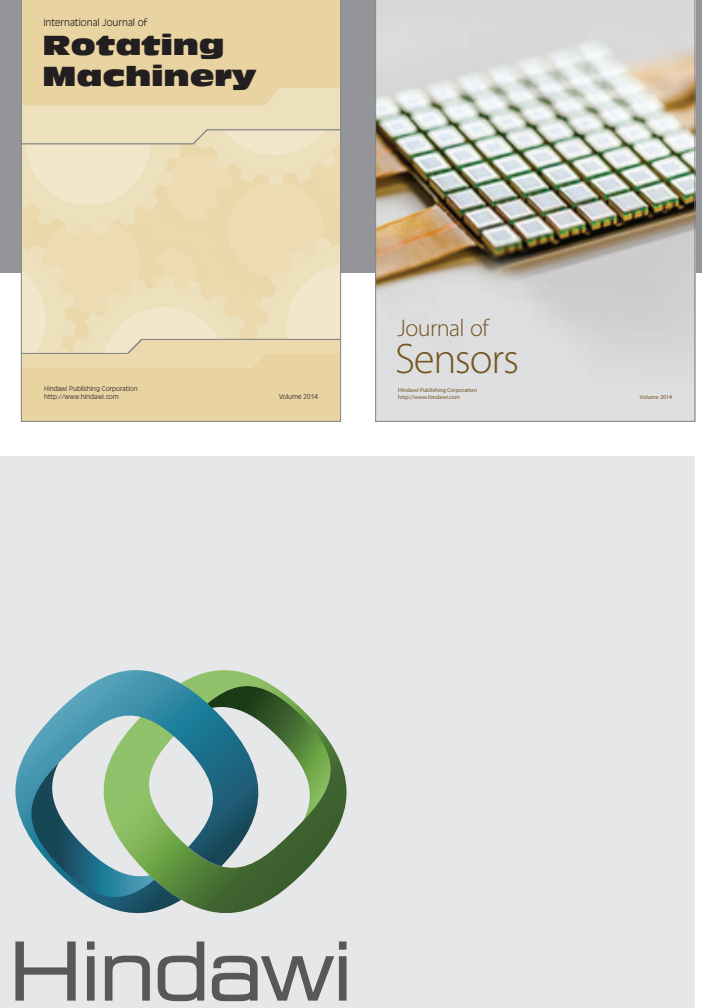

Submit your manuscripts at http://www.hindawi.com
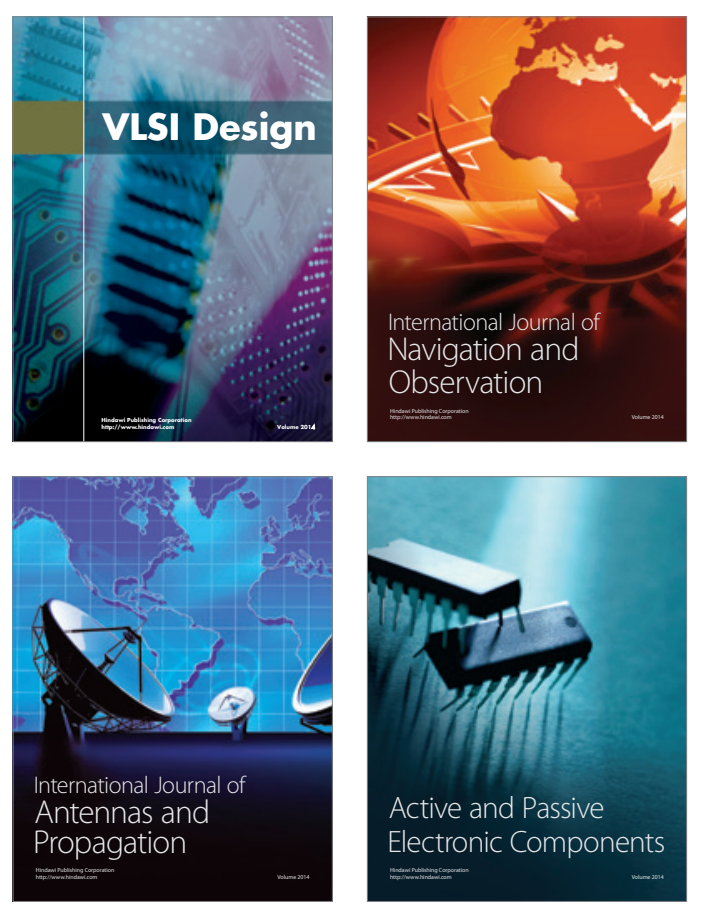
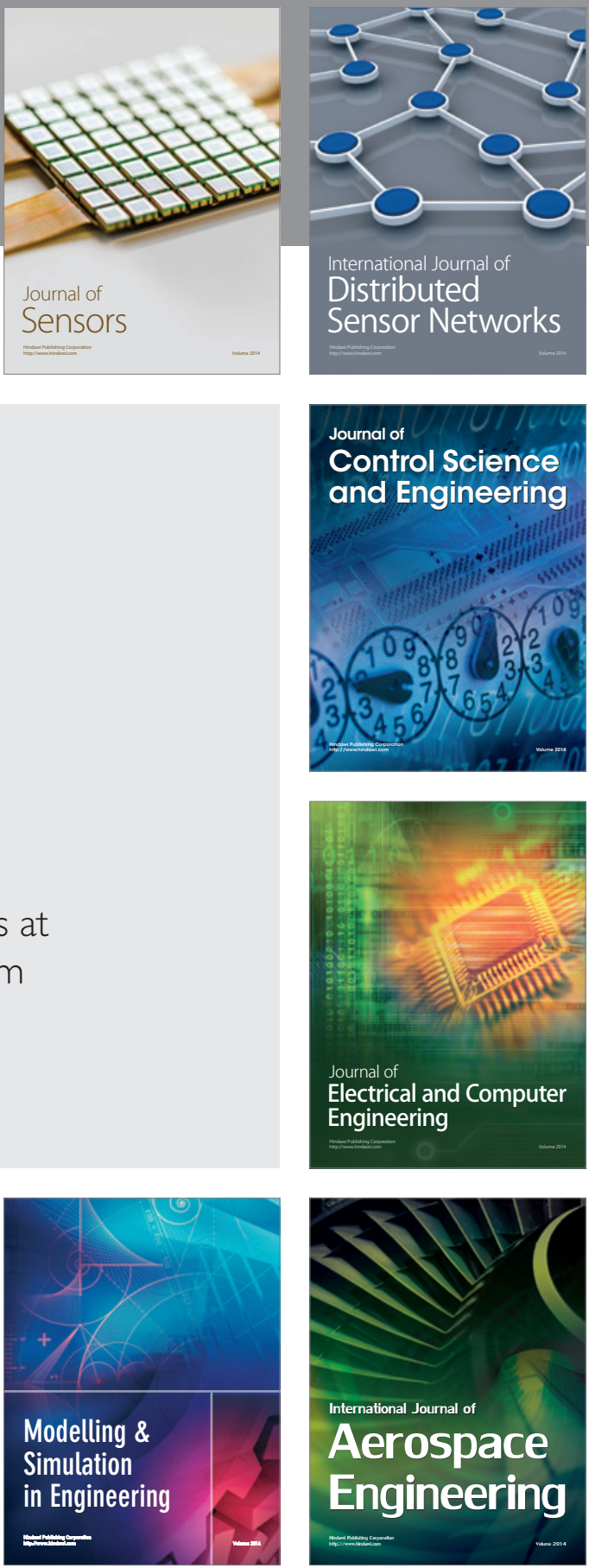

Journal of

Control Science

and Engineering
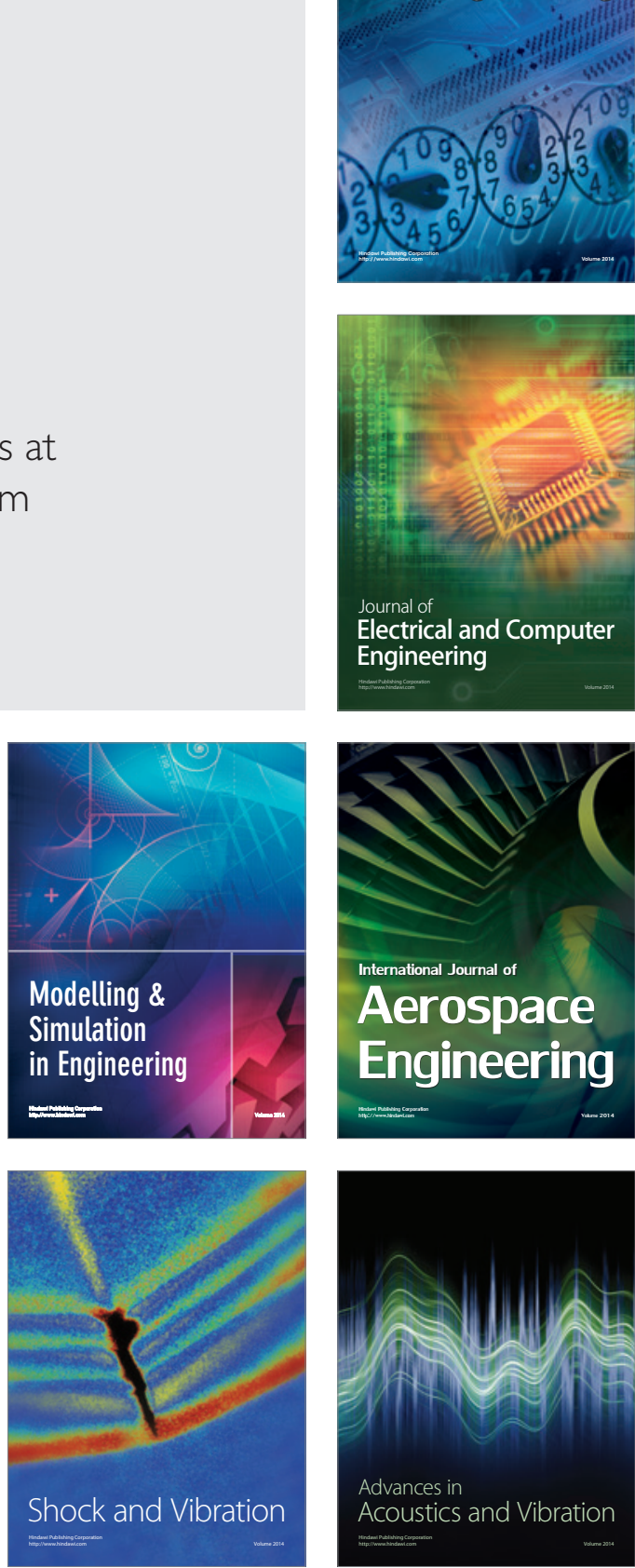